\title{
Actualización en patología trofoblástica gestacional: mola hidatiforme y neoplasias
}

Update in gestational trophoblastic pathology: hydatidiform mole and neoplasias

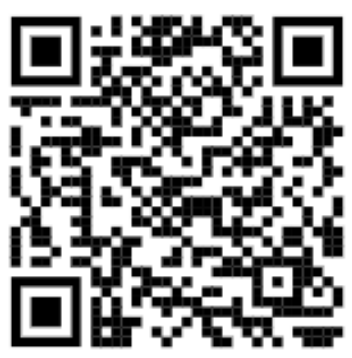

RECIBIDO

$17 / 03 / 2019$

\author{
${ }^{1}$ Dra. Andrea Espinoza Artavia \\ Investigadora independiente, San José, Costa Rica \\ a.espinozaartavia@gmail.com. \\ https://orcid.org/0000-0001-5512-2177 \\ ${ }^{2}$ Dra. Roxana Fernández Vaglio \\ Investigadora independiente, San José, Costa Rica \\ rox.fernandez.vaglio@gmail.com \\ https://orcid.org/0000-0003-2780-7560 \\ ${ }^{3}$ Dra. Thania Solar Del Valle \\ Investigadora independiente, San José, Costa Rica \\ thania.solar@gmail.com \\ https://orcid.org/0000-0001-5693-8725
}

CORREGIDO

ACEPTADO

$30 / 03 / 2019$

$08 / 04 / 2019$

\section{RESUMEN}

La enfermedad trofoblástica gestacional está compuesta por una gama de patologías poco comunes asociadas al embarazo, que van desde benignas hasta malignas. Es importante realizar un diagnóstico oportuno mediante el uso de ultrasonido; apoyándose con estudios de laboratorio y gabinete, estudios histológicos y moleculares. En términos generales, el tratamiento de la enfermedad molar premaligna va a consistir en la evacuación uterina. El uso de quimioterapeúticos y de intervenciones quirúrgicas, en conjunto con el uso de

${ }^{1}$ Médico general, graduada de la Universidad de Costa Rica tI (UCR). Código médico: 15174 a.espinozaartavia@gmail.com.

${ }^{2}$ Médico general, graduada de la Universidad de Costa Rica (UCR). Código médico: 15160 rox.fernandez.vaglio@gmail.com.

${ }^{3}$ Médico general, graduada de la Universidad de Costa Rica (UCR). Código médico: 15161 thania.solar@gmail.com radioterapia, podría llegar a tener un papel importante en el tratamiento tanto de estas como de las enfermedades trofoblásticas neoplásicas. La hormona gonadotropina coriónica humana es un biomarcador crucial para medir la progresión, respuesta a tratamiento y seguimiento de estas enfermedades. Gracias a la implementación de mejoras en el diagnóstico y tratamiento, la presentación clínica y morbilidad de las enfermedades ha cambiado conforme pasan los años.

PALABRAS CLAVE: mola hidatiforme; enfermedad trofoblástica gestacional; neoplasias trofoblásticas; coriocarcinoma; tumor trofoblástico localizado en la placenta. 


\begin{abstract}
Gestational trophoblastic disease is composed of a range of rare pathologies associated with pregnancy, ranging from benign to malignant. It is important to make an opportune diagnosis through the use of ultrasound; supported with laboratory and imaging studies, histological and molecular studies. In general terms, the treatment of pre-malignant molar disease will consist of uterine evacuation. The use of chemotherapeutic and surgical interventions, together with the use of radiotherapy, could have an important role in the treatment of this entities as well as for the neoplastic trophoblastic diseases. The hormone human chorionic gonadotropin is a crucial biomarker to measure the progression, response to treatment and follow-up of these diseases. Thanks to the implementation of improvements in diagnosis and treatment, the clinical presentation and morbidity of the diseases has changed as the years go by.
\end{abstract}

KEYWORDS: hydatidiform mole; gestational trophoblastic disease; trophoblastic neoplasms; choriocarcinoma; trophoblastic tumor, placental site.

\section{INTRODUCCIÓN}

La enfermedad trofoblástica gestacional (ETG) se origina de tejido placentario anormal y consiste en una serie de patologías asociadas al embarazo que se pueden dividir en benigna, premaligna y maligna. En condiciones normales, el tejido trofoblástico sano invade el endometrio y desarrolla una rica vasculatura, lo que eventualmente se conformaría en lo que conocemos como placenta.

En la ETG los mecanismos de regulación fallan, lo que produce tumores altamente invasivos, metastásicos y muy vascularizados (1). Esta patología es más común en Asia y Latinoamérica (2). Histológicamente, incluye las formas premalignas como la mola hidatiforme parcial y completa, así como el conjunto de neoplasias trofoblásticas gestacionales (NTG), entre las cuales se encuentran la mola invasiva, el coriocarcinoma, el tumor trofoblástico de sitio placentario (TTSP) y el tumor trofoblástico epitelioide (TTE). Actualmente, con la mejoría y avance de las estrategias diagnósticas, terapéuticas y de seguimiento, la mayoría de mujeres afectadas por estas patologías pueden alcanzar la curación si se realiza un manejo adecuado (3). Al ser una patología más común en nuestro medio, esta revisión se basa en bibliografía reciente para poder ser implementada por profesionales de salud y promover el diagnóstico temprano de estas patologías.

Asimismo, se espera descubrir los faltantes en el conocimiento e investigación actual, a manera de motivar a todos los profesionales a seguir realizando aportes para el manejo de las mismas. 


\section{MATERIALES Y MÉTODOS}

Para la elaboración de esta revisión, se buscó bibliografía reciente en Pubmed con los términos "enfermedad", "trofoblástica" y "gestacional". La búsqueda se enfocó en asociar dichos términos. Se tomó en cuenta el uso de bibliografía tanto en inglés como en español. Se dará prioridad a la patología premaligna y maligna, por lo que la patología benigna no es realmente de interés para esta revisión.

\section{PATOLOGÍA MOLAR PREMALIGNA: MOLA HIDATIFORME PARCIAL Y COMPLETA}

\section{- Epidemiología}

La incidencia de mola hidatiforme presenta gran variabilidad según la localización geográfica, pero en la mayoría del mundo la incidencia va a ser de aproximadamente 1 por cada 1000 embarazos (4). Sin embargo, a nivel mundial ha ocurrido una disminución gradual en la incidencia de esta patología, que se estima podría deberse a cambios nutricionales. Aproximadamente el $80 \%$ de las ETG serán por mola hidatiforme (5).

\section{- Factores de riesgo}

Los dos factores de riesgo clínicos más importantes para desarrollar un embarazo molar, son los extremos de edad reproductiva en las mujeres (<15 o >45 años) y el antecedente de un embarazo molar previo. El riesgo en mujeres menores de 15 años es hasta 20 veces mayor. Posteriormente, la incidencia vuelve a aumentar de forma progresiva a los 35 años, con 5-10 veces más riesgo luego de los 45 años de presentar mola, y un riesgo 200 veces mayor en mujeres con 50 años o más respecto a mujeres entre los 20 y 35 años. Esto al mismo tiempo se asocia a un incremento en la necesidad de tratamientos quimioterapéuticos posteriores $(3,6,7)$.

El riesgo de desarrollar un segundo embarazo molar tras uno previo es de 1$2 \%$, mientras que el riesgo de un tercer embarazo molar tras dos embarazos molares previos es de hasta un $25 \%$. A los pacientes que presentan estos factores de riesgo, se les debe realizar un ultrasonido durante el primer trimestre para evaluar la cavidad uterina (6).

Un estudio transversal publicado en el 2016, con 255 embarazos molares y más de 105.000 nacimientos vivos, demostró que la etnia o raza es un factor de riesgo determinante para el desarrollo de una mola, ya sea completa o parcial. En dicho estudio las mujeres asiáticas presentaron el mayor riesgo de desarrollar una mola completa, en comparación a mujeres blancas, hispanas y afroamericanas. Las mujeres blancas presentaron el mayor riesgo de desarrollar mola parcial (8).

El uso de píldoras anticonceptivas también se ha visto relacionado con un aumento en el riesgo de embarazo molar, el cual aumenta progresivamente de acorde al tiempo de consumo de las mismas (2).

También existe una asociación entre el desarrollo de mola completa con la deficiencia de caroteno (3). La menarca tardía, el flujo menstrual ligero, la paridad, el grupo sanguíneo, la edad paternal, el fumado y el consumo de alcohol han mostrado asociaciones en ciertos estudios, sin embargo, su relación aún es poco clara (2). 
Estudios genéticos recientes han demostrado mutaciones a nivel de cromosomas, (NLRP7 en el cromosoma $19 q 13$ y KHDC3L en el cromosoma 6), que se han visto implicadas en formas familiares recurrentes de mola hidatiforme. Esta forma familiar consiste en un desorden autosómico recesivo causante de molas completas (2).

\section{- Patogénesis}

Las molas hidatiformes se producen por una gametogénesis y fertilización anormal, de forma más frecuente en los extremos de la vida reproductiva. La progresión de la mola hidatiforme completa y parcial hacia una NTG ocurrirá en un $15-20 \%$ y un $0,5-5 \%$ respectivamente (3).

Un $80-90 \%$ de las molas completas son el resultado de la fertilización de un óvulo vacío por un espermatozoide haploide, el cual posteriormente duplica sus cromosomas. Citogenéticamente las molas completas son diploides, y un $95 \%$ tienen un patrón cromosómico paternal homólogo 46 XX (es decir, con ambas "X" provenientes de descendencia paterna). En el resto de los casos, un óvulo vacío es fertilizado por 2 espermatozoides haploides, lo que resulta en un cariotipo $46 \mathrm{XX}$ o $46 \mathrm{XY}(2,3,6)$. En el caso de las molas parciales, un óvulo haploide es fertilizado por 2 esperamatozoides. La mayoría de molas parciales presentan un cariotipo triploide, de descendencia tanto paterna como materna, resultando en un cariotipo $69 \mathrm{XXY}, \mathrm{XXX}$ y en pocos casos $\mathrm{XYY}(2,6)$.

La mola completa consiste en vellosidades hidrópicas o vesículas semitransparentes de distintos tamaños, en ausencia de tejido placentario normal. Al inicio pueden tener mínimos o nulos cambios a nivel de vellosidades. Histológicamente, las molas completas tienen formación florida de cisternas, proliferación trofoblástica y ausencia de partes fetales. La atipia citológica y las figuras mitóticas son también comunes. En el primer trimestre, las vellosidades pueden tener una apariencia polipoide distintiva con estroma anormal y pequeños cambios de hiperplasia. Estos cambios histológicos son menos marcados en la mola parcial. A diferencia de la mola completa, la mola parcial presentará partes o células fetales (3).

Un inhibidor de quinasas ciclinadependiente p57 está ausente en las molas completas debido a la falta de genoma materno, mientras que en las molas parciales estará presente. Sin embargo, con esto no se puede diferenciar una mola parcial de un embarazo no-molar (3).

\section{- Clínica}

La presentación clínica usual en hasta $46 \%$ de los casos de mola completa es el sangrado vaginal, generalmente entre la semana 6-16 de gestación. El crecimiento uterino mayor de lo esperado para la edad gestacional se presentará en aproximadamente un $24 \%$ de los casos. Al igual que con molas completas, las molas parciales debutan como un sangrado vaginal en un $75 \%$ de los casos, sinembargo esto suele darse de forma aún más tardía (9).

Debido a la mejoría en el diagnóstico temprano durante el primer trimestre con métodos ultrasonográficos, muchas molas se presentarán asintomáticas a la hora de realizar el diagnóstico. Asimismo, complicaciones clínicas tales como la hiperémesis gravídica, la preeclampsia, los síntomas de hipertiroidismo, los 
tromboembolismos y la presencia de grandes quistes teca-luteínicos, se observan con menor frecuencia en la actualidad. En caso de que se dé la evacuación uterina, se pueden observar vesículas en el tejido expulsado. En muchos casos, los embarazos molares pueden ser diagnosticados solo por histología debido a la similitud que presentan con los abortos (2, 3, 9).

\section{- Diagnóstico}

Ante la sospecha de enfermedad trofoblástica gestacional, se debe realizar una historia clínica exhaustiva que incluya fechas, duración y desenlace de embarazos anteriores, antecedente de embarazo molar y demás antecedentes ginecoobstétricos y quirúrgicos. Asimismo, se debe realizar un examen físico completo, teniendo en cuenta la posibilidad de presencia de metástasis.

Se debe realizar una prueba cuantitativa de hCG. En caso de mola hidatiforme, los niveles de hCG pueden ser superiores a los de un embarazo normal, usualmente mayores a 100,000 $\mathrm{mUl} / \mathrm{mL}$. Los niveles de hCG marcadamente elevados son más comunes en molas hidatiformes completas que en las parciales. Además de los niveles de hCG, las molas parcial y completa pueden diferenciarse mediante su morfología macroscópica, cariotipo, apariencia histológica y manifestaciones clínicas.

En caso de sospecha de ETG se debe realizar un ultrasonido transvaginal. La mola completa puede diagnosticarse por características como: embarazo anembriónico, ausencia de líquido amniótico, masa central heterogénea con numerosos espacios anecoicos en apariencia de "tormenta de nieve" y quistes ováricos tecaluteínicos. Estos hallazgos son más evidentes después del segundo trimestre de gestación (2).

Se debe pensar en la posibilidad de un embarazo molar con mola parcial o incompleta cuando se encuentra una placenta con grosor mayor al normal, con espacios quísticos y con ecogenicidad de vellosidades coriónicas. Asimismo, se puede encontrar un feto que podría ser viable. En este tipo de mola se puede observar una cantidad de líquido amniótico menor que la normal para embarazos de la misma edad gestacional, además una relación entre el diámetro transverso y el antero-posterior de más de $1.5(2,10)$.

En general, el ultrasonido como método diagnóstico de embarazo molar presenta sensibilidad y especificidad relativamente bajas, por lo que se recomienda realizar siempre un análisis histológico. Este tipo de análisis también se debería efectuar después de cada embarazo no viable para descartar enfermedad trofoblástica gestacional (2).

Por otra parte, ciertas técnicas citogenéticas, como el bandeo cromosómico o los polimorfismos de longitud de fragmentos de restricción (RFLP), han permitido analizar el ADN y encontrar patrones cromosómicos que permiten identificar y diferenciar molas hidatiformes completas e incompletas (5).

\section{- Tratamiento}

Si se desea conservar la posibilidad de fertilidad, el método de elección para la evacuación de un embarazo molar es la evacuación uterina mediante succión y curetaje, independientemente del tamaño uterino. 
Se recomienda iniciar una infusión de oxitocina al comenzar el procedimiento, y mantenerla posteriormente por varias horas para mejorar la contractilidad uterina, con el fin de promover la evacuación de restos de tejido y disminuir el sangrado. El riesgo de hemorragia aumenta con las semanas de embarazo, por lo que si la paciente presenta un tamaño uterino correspondiente a 16 semanas de embarazo o más, se recomienda tener a disposición componentes sanguíneos en caso de necesidad de transfusión. Se le debe administrar inmunoglobulina anti-D a las mujeres $\mathrm{Rh}$ negativas a la hora del procedimiento, debido a que el factor $\mathrm{RhD}$ se expresa en el trofoblasto. Para realizar la aspiración, se recomienda una cánula de succión de 12-14mm. Con el fin de que el procedimiento sea exitoso, se recomienda el uso de equipo y técnicas adecuadas, acceso rápido a productos sanguíneos, monitoreo constante durante el procedimiento y posterior a este, y el reconocimiento temprano de complicaciones y la corrección inmediata de las mismas. En caso de no presentar sangrado persistente después del procedimiento, generalmente no es necesario realizar una segunda evacuación (3).

Si la mujer presenta paridad satisfecha, la histerectomía es un procedimiento alternativo, ya que además de la evacuación del embarazo molar, disminuye la necesidad de quimioterapia subsecuente al eliminar el riesgo de invasión miometrial en caso de enfermedad trofoblástica persistente (3). No se recomienda la inducción farmacológica de la labor, ya que esta podría aumentar el riesgo de contracción miometrial y embolismo tumoral mediante el sistema venoso. Asimismo, puede incrementar el riesgo de aborto incompleto y la posibilidad de desarrollo de neoplasia trofoblástica postmolar con requerimiento de quimioterapia (2).

En los casos en que el riesgo de neoplasia trofoblástica gestacional es mayor que el normal, o que por alguna razón no sea posible un seguimiento adecuando de la paciente, se puede administrar metotrexate o actinomicina $D$ de manera profiláctica después de la evacuación del embarazo molar, ya que su uso ha sido asociado con una reducción de la incidencia de neoplasia trofoblástica gestacional (3).

\section{- Seguimiento}

A todas las pacientes con diagnóstico de embarazo molar se les debe realizar tamizaje completo para determinar la extensión de la enfermedad. Entre los exámenes recomendados se encuentran: medición cuantitativa de hCG, hemograma completo, pruebas de coagulación, pruebas de función hepática, pruebas de función renal, electrolitos, grupo sanguíneo y $\mathrm{Rh}$, perfil tiroideo, serologías por $\mathrm{VIH}$ y hepatitis $\mathrm{B}$, radiografía de tórax y ultrasonido pélvico (2).

Según las recomendaciones de FIGO, a las pacientes con embarazo molar se les debe dar seguimiento con mediciones semanales de hCG hasta dos semanas después de que esta se normalice. Posteriormente, se deben realizar mediciones mensuales por dos a seis meses, y después, cada dos meses por seis meses más (2).

A todas las pacientes se les debe recomendar utilizar algún método anticonceptivo durante al menos seis 
meses después de que los niveles de hCG se normalicen (2).

\section{PATOLOGÍA MALIGNA}

Pese a que estos tumores abarcan menos del $1 \%$ de los tumores ginecológicos, representan un riesgo importante en mujeres de edad reproductiva (1). Las NTG están asociadas en un $25 \%$ con antecedente de aborto, en $5 \%$ con embarazo ectópico, en $20 \%$ con embarazo a término y en un $50 \%$ con mola hidatiforme. Estudios restrospectivos mostraron que la edad materna, los antecedentes de embarazo molar y niveles de hCG elevados posteriores a la evacuación uterina, están asociados a un mayor riesgo de presentar NTG (2).

Las NTG pueden ser metastásicas o nometastásicas (11).

Pueden cursar de forma asintomática, como un sangrado vaginal anormal, o como sangrados o síntomas y signos provenientes de los sitios metastásicos, tales como cefalea, convulsiones, disnea, tos y dolor de pecho $(3,9)$.

Los TTSP y TTE suelen valorarse y clasificarse como entidades por aparte, ya que tienen una presentación clínica y patológica diferente a la de las demás neoplasias (2).

\section{MOLA INVASIVA}

Consiste en la invasión del tejido molar hacia el miometrio (6).

Suelen darse inmediatamente posterior al embarazo que los precede (9).

\section{- Epidemiología}

Se estima que tiene una incidencia de un $5-10 \%$ en la población (6).
Aproximadamente $10 \%$ de las mujeres con molas hidatiformes presentarán este tipo de neoplasia. Estas comprenden aproximadamente el $15 \%$ de todas las ETG $(5,12)$.

\section{- Patogénesis}

Histológicamente se asemejan a las molas completas, con células de citotrofoblasto y sincitiotrofoblasto hiperplásicas, marcada atipia celular, y vellosidades coriónicas más irregulares y atípicas en comparación a las molas completas (7).

\section{- Clínica}

La hemorragia en estos casos puede ser severa y el sangrado puede ser tanto vaginal como intraperitoneal (6).

\section{CORIOCARCINOMA}

\section{- Epidemiología}

En Norteamérica y Europa tienen una incidencia aproximada de 1 en 40.00050.000 embarazos. Un $25 \%$ ocurre posterior a abortos, $24 \%$ posterior a embarazos de término y el resto luego de embarazos molares $(2,3)$. Se estima que este tumor comprende aproximadamente el $5 \%$ de todas las NTG (5). El coriocarcinoma junto con el TTSP y el TTE suelen darse meses o años posterior al embarazo precedente (9). Sin embargo, usualmente el periodo de latencia es menor para el coriocarcinoma y mayor para el TTE (13).

\section{- Patogénesis}

El tumor es grande, con áreas necróticas y hemorrágicas. Histológicamente carece de vellosidades coriónicas, y presenta células de trofoblasto y citotrofoblasto intermedio, rodeados de sincitiotrofoblasto con áreas necróticas y 
hemorrágicas. Presenta un cariotipo complejo, siendo la mayoría conformado por un patrón cromosómico XX (3). La principal ruta de metástasis será por vía hematógena (9).

\section{- Clínica}

En la mayoría de los casos, la presentación usual corresponde a sangrado vaginal (13). Típicamente, el coriocarcinoma se puede confundir con otras enfermedades, debido a que las metástasis hemorrágicas pueden producir hematuria, hemoptisis, hematoquesia, accidentes cerebrovasculares o sangrado vaginal. Algunos lugares comunes de metástasis son: pulmón (60-95\%), vagina (40-50\%), vulva o cérvix (10-15\%), cerebro (5-15\%), hígado (5-15\%), trompas uterinas, ovarios, riñón, bazo u otras vísceras gastrointestinales $(3,6)$.

\section{TUMOR TROFOBLÁSTICO DEL SITIO PLACENTARIO (TTSP)}

\section{- Epidemiología}

Este tumor es poco frecuente, con aproximadamente solo 100 casos reportados en la literatura. Sin embargo, tiene el potencial agresivo de producir metástasis y causar la muerte, siendo la mayor causante de mortalidad entre todas las ETG $(4,5)$. Aproximadamente el $60 \%$ se presentará posterior a un embarazo de término, al igual que en el caso de los tumores trofoblásticos epitelioides.

El resto se presentará posterior a abortos o molas (9). Los nódulos atípicos de sitio placentario, los cuales se encuentran dentro del espectro benigno de las enfermedades trofoblásticas gestacionales, pueden coexistir o transformarse en TTSP o TTE en un 10$15 \%$ de los casos (14). Menos del $1 \%$ de pacientes con ETG presenta esta variante, la cual corresponde a un $0.1-2 \%$ de todas las NTG $(2,6)$.

\section{- Patogénesis}

El tumor tiene una apariencia blancoamarillenta, con masas nodulares de 1-10 $\mathrm{cm}$ en endometrio, y hasta en la mitad de los casos, en miometrio. Puede estar bien circunscrito, pero en ocasiones puede tener una forma polipoide que se proyecta hacia la cavidad uterina. Puede inclusive extenderse hacia serosa o anexos, y en ocasiones hacer metástasis a distancia $(3,4,13)$.

Histológicamente, carece de vellosidades coriónicas y se forma a partir de células mononucleares de trofoblasto intermedio. Presenta células con membranas nucleares irregulares, núcleos hipercrómicos y citoplasma denso eosinofílico, con pocas células mitóticas. Secreta varios biomarcadores de forma difusa, entre ellos el lactógeno placentario humano. A diferencia de las otras NTG anteriormente vistas, presenta una expresión focal de hCG. Presenta un cariotipo con extraños desequilibrios $(3,4$, 13). La principal ruta de metástasis será por vía linfática, al igual que en el caso de los tumores trofoblásticos epitelioides (9).

\section{- Clínica}

Usualmente se presenta como un sangrado vaginal persistente, amenorrea o aumento del tamaño uterino.

También puede debutar mediante síndrome nefrótico, virilización y síntomas paraneoplásicos, sinembargo esto es poco común (2).

TUMOR TROFOBLÁSTICO EPITELIOIDE (TTE)

\section{- Epidemiología}

Corresponde a $<1 \%$ de las NTG, con 
aproximadamente solo 90 casos publicados en la literatura (7).

\section{- Patogénesis}

El tumor tiene una apariencia marrón, con masas nodulares discretas o quistes hemorrágicos que invaden profundamente los tejidos adyacentes. Aproximadamente la mitad aparece en el cérvix o segmento inferior del útero, por lo cual suele ser confundido con carcinoma de cérvix. En algunos casos aparece en fondo 0 ligamento ancho. Histológicamente se forma a partir de trofoblasto intermedio tipo coriónico, en conformaciones de islas con cantidades moderadas de citoplasma eosinofílico, rodeadas de necrosis extensa y matriz hialina $(2,3)$.

\section{- Clínica}

Usualmente presentan una sintomatología similar a la del TTSP, en $70 \%$ de los casos se presentan como un sangrado vaginal anormal. Este tumor puede coexistir con otras NTG como coriocarcinoma o TTSP (2).

\section{- Diagnóstico}

El diagnóstico de una NTG se puede realizar en el seguimiento posterior al tratamiento de una ETG. Por su parte, el diagnóstico en las pacientes que no han presentado un embarazo molar puede ser más complicado, ya que no es rutina la realización de valoraciones de hCG luego de los mismos. En estas pacientes se debe sospechar la enfermedad ante sangrados vaginales anormales, sangrados de sitios metastásicos (por ejemplo hemoptisis), síntomas pulmonares y neurológicos (cefalea, convulsiones, hemiparesia) $(3,15)$.
Existen criterios diagnósticos para las NTG definidos por FIGO en 2018. Los mismos son (3):

- Meseta en la medición de hCG luego de 3 semanas o más del tratamiento de una ETG.

- Aumento de en los valores de hCG durante 3 mediciones consecutivas semanales en un periodo de 2 o más semanas.

- Diagnóstico histológico de coriocarcinoma.

Se ha visto que, en las ETG, la beta hCG puede encontrarse en fragmentos, por lo que resulta necesario que la prueba utilizada para la detección sea capaz de identificarlos todos. Muchos de los ensayos utilizados no detectan todos los fragmentos o son muy sensibles a ciertos de ellos, lo que puede originar falsos negativos o positivos. (16). También se debe tomar en cuenta que ciertas pacientes (por ejemplo, mujeres perimenupáusicas), pueden presentar anticuerpos heterófilos, lo que puede significar un falso positivo (17).

Una vez que se detecten alteraciones de los niveles de hCG, es necesaria la evaluación de la paciente para el reconocimiento de posibles metástasis. En aquellas pacientes con alteraciones persistentes en hCG, es importante la realización de ultrasonido pélvico, en el que es posible valorar masas focales no específicas con componente endometrial variable, distintos grados de vascularidad, y variable invasión endometrial (9). Se puede utilizar la radiografía de tórax para la detección de metástasis pulmonares, ultrasonido o tomografía para la detección de afectación hepática, y resonancia magnética o tomografía para evaluación

\section{2}

Revista Médica Sinergia Vol. 4 (5), Mayo 2019

ISSN:2215-4523 / e-ISSN:2215-5279

http://revistamedicasinergia.com 
cerebral (3). Otros estudios que se deben solicitar son hemograma, pruebas de función renal y hepática, pruebas de coagulación y factor Rh (18).

\section{ESTADÍOS FIGO, CLASIFICACIÓN Y SISTEMA DE PUNTAJE DE LA OMS}

El régimen de tratamiento establecido dependerá del estadio, la clasificación y el puntaje de riesgo de la NTG. Típicamente, esto se realiza usando una combinación de lo propuesto por la Federación Internacional de Ginecología y Obstetricia (FIGO), y la Organización Mundial de la Salud (OMS o WHO por sus siglas en inglés). Un riesgo de 6 o menor según el sistema de puntaje de la OMS se considera como de bajo riesgo, mientras que uno superior a 6 será de alto riesgo. Todos los pacientes deberán ser clasificados con un estadio y un puntaje (por ejemplo: estadio II:5) (3).

La FIGO clasifica y estratifica las NTG de la siguiente forma $(2,3)$ :

- Estadio I: tumores trofoblásticos gestacionales estrictamente confinados al cuerpo uterino.

- Estadio II: tumores trofoblásticos gestacionales que se extienden a anexo, ligamento ancho, o a vagina, pero limitado a estructuras genitales.

- Estadio III: tumores trofoblásticos gestacionales que se extienden a pulmones, con o sin extensión a estructuras genitales.

- Estadio IV: todos los otros sitios metastásicos.

El sistema de puntación de la OMS utiliza las siguientes variables: edad $(<40: 0$,
$>40: 1$ ), embarazo precedente (mola:0, aborto:1, término:2), intervalo desde último embarazo en meses (<4: 0, 4-6: 1 , 7-12: 2, >12: 4), nivel de hCG sérico $(\mathrm{Ul} / \mathrm{mL})$ previo al tratamiento $(<103: 0$, $>103-<104$ : 1,>104-<105: 2, >105: 4), tamaño tumoral superior en centímetros (3-4: 1, 25:2), sitio de metástasis (pulmón: 0 , bazo y riñón: 1 , tracto gastrointestinal: 2, cerebro e hígado: 4), número de metástasis (1-4: 1, 5-8: 2, >8: 4) y fallo al tratamiento quimioterapéutico previo (una sola droga: 2, dos o más drogas: 4) (3, 19).

\section{TRATAMIENTO}

El manejo de estas pacientes depende en gran medida de la evaluación de riesgo realizada de acuerdo a factores pronósticos.

\section{- NTG de bajo riesgo}

La mayoría de las pacientes, cerca de $95 \%$, se clasifican como de riesgo bajo (20). Las pacientes de bajo riesgo (puntajes menores de 7 o estadio I) se pueden dividir en dos grupos. Aquellas pacientes con valores de 0-4 pueden ser tratadas con una sola droga (3). Sin embargo, para las pacientes con valores de 5 ó 6 , se ha visto que solo $30 \%$ tienen respuesta a monoterapia, por lo que deben recibir múltiples quimioterapéuticos (15). En las pacientes con diagnóstico de coriocarcinoma también se debe considerar el uso de múltiples drogas (3). En las pacientes en estadio I se puede considerar histerectomía en aquellas perimenopáusicas con paridad satisfecha, con lo cual se puede reducir el número de ciclos de quimioterapia necesarios (20). Las drogas quimioterapéuticas de primera línea en el tratamiento de la enfermedad de bajo riesgo son el metotrexato y la 
actinomicina $D$ (21). De acuerdo con un meta-análisis realizado en 2016, se ha visto que la actinomicina $D$ puede llevar a mayores tasas de curación que el metotrexato como monoterapia en las GTN de bajo riesgo (22). Pese a lo anterior, se ha demostrado que sin importar la respuesta inicial al primer tratamiento se pueden utilizar otros fármacos, con lo que se logra cerca de $100 \%$ de remisión, por lo que debido a la menor toxicidad observada con metotrexato se suele preferir esta droga como primera línea de tratamiento (20). En lo casos en los que se observe respuesta inadecuada, toxicidad 0 mesetas/aumentos en hCG en dos semanas consecutivas (resistencia primaria), se debe considerar el cambio de la droga inicial $(3,20)$.

En las pacientes que presenten resistencia o recurrencia pese al cambio a monoterapia de segunda línea, se debe utilizar terapia con combinación de drogas (20).

En un estudio realizado en 2016 se intentó encontrar posibles factores predictivos de recaída luego del tratamiento con metotrexato.

Dentro de los hallazgos, se vio que las pacientes con una NTG posterior a un parto y aquellas que requirieron más de 4 cursos de metotrexato, tenían mayor riesgo de recurrencia (23).Los distintos esquemas de tratamiento para las pacientes con clasificadas de bajo riesgo se describen a continuación:

\section{Metotrexato}

- 50mg intramuscular cada $48 \mathrm{~h}$ (días 1,3,5,7) con administración de $15 \mathrm{mg}$ de ácido folínico oral cada 24 h-30h (días 2,4,6,8). Esto se repite cada 2 semanas $(3,20)$. Se obtiene $74-90 \%$ de remisión (15).

○ $\quad 0.3-0.5 \mathrm{mg} / \mathrm{Kg}$ (máximo $25 \mathrm{mg}$ por dosis) intramuscular o intravenoso por 5 días, igual que el esquema anterior se repite cada 2 semanas (20). Se ha observado $87-93 \%$ de remisión con este esquema (15).

- 30-50mg/m2 intramuscular cada semana (3), un 49-74\% logra remisión (15).

- 100mg/m2 en infusión IV en 30 minutos para luego pasar $200 \mathrm{mg} / \mathrm{m} 2$ en $12 \mathrm{~h}$. Se administran $15 \mathrm{mg}$ de ácido folínico cada $12 \mathrm{~h}$ ( 6 dosis) luego de $24 \mathrm{~h}$ de iniciado el tratamiento con metotrexato (20), con remisión en $69-90 \%$ (15).

\section{Actinomicina D}

- Pulso intravenoso de $1.25 \mathrm{mg} / \mathrm{m} 2$ cada 2 semanas (3), con remisión de $69-90 \%$ (15).

- $0.5 \mathrm{mg}$ intravenoso por 5 días cada 2 semanas (3), se observa remisión en $77-94 \%$ de las pacientes (21).

El tratamiento se continúa hasta obtener mediciones indetectables de hCG, y posteriormente se debe continuar con 2-3 cursos más (consolidación) para disminuir las probabilidades de recaída $(3,15,20$, 21).

\section{- NTG de alto riesgo}

Como se explicó previamente, en este grupo se encuentran las pacientes con puntajes mayores a 6 (20). Para el tratamiento de estas pacientes, se han desarrollado diversas combinaciones de tratamiento, sin embargo el más utilizado es el denominado por sus siglas en inglés 
como EMA-CO $(3,20)$. Se ha visto que en paciente con enfermedad metastásica de alto riesgo, la histerectomía primaria podría contribuir a la cura o al menos a limitar las dosis de quimioterapia utilizada (24).

A continuación, se describe el esquema EMA-CO (etopósido, metotrexato, actinomicina D, ciclofosfamida y vincristina):

Cada semana se alterna el régimen utilizado (3).

- Primera semana.

- Día 1:

- Etopósido: $100 \mathrm{mg} / \mathrm{m} 2$ en infusión intravenosa a pasar en 30 minutos $(3,20)$.

- Actinomicina D: $0.5 \mathrm{mg}$ en bolo intravenoso $(3,20)$.

- Metotrexato: $100 \mathrm{mg} / \mathrm{m} 2$ en bolo intravenoso, seguidos de $200 \mathrm{mg} / \mathrm{m} 2$ en infusión intravenosa a pasar en 12h(3).

- Día 2:

- Etopósido: $100 \mathrm{mg} / \mathrm{m} 2$ en infusión a pasar en 20 minutos (3).

- Acido folínico: 15mg intramuscular u oral cada $12 \mathrm{~h}$ a empezar $24 \mathrm{~h}$ luego de iniciada la infusión de metotrexato, por 4 dosis $(3,20)$.

- Actinomicina D: $0.5 \mathrm{mg}$ en bolo intravenoso (20).

- Segunda semana.

- Día 8:

- Vincristina: $0.8 \mathrm{mg} / \mathrm{m} 2 \quad(2 \mathrm{mg}$ como máximo) en bolo intravenoso $(3,20)$.

- Ciclofosfamida: $600 \mathrm{mg} / \mathrm{m} 2$ en infusión intravenosa a pasar en 30 minutos $(3,20)$.

En este grupo de pacientes, el tratamiento se debe de continuar por 6 semanas luego de la normalización de valores de hCG; en el caso de pacientes con metástasis hepáticas 0 cerebrales se podría continuar hasta por 8 semanas (20).

Existe un subgrupo de mujeres con muy alto riesgo, aquellas con puntajes mayores o iguales a $13(3,20)$. Se ha visto que ellas tienen peor pronóstico, además de que pueden presentar quimioresistencia y complicaciones (20). En estas pacientes se ha visto beneficio en iniciar con dosis bajas de etopósido $(100 \mathrm{mg} / \mathrm{m} 2)$ y cisplatino $(20 \mathrm{mg} / \mathrm{m} 2)$ en días 1 y 2 cada semana, esto por 1-2 semanas $(3,20)$.

Precisamente, en estos casos de muy alto riesgo y en aquellas pacientes con metástasis hepáticas o cerebrales, se podría considerar el cambio de régimen de primera línea de tratamiento. En lugar de utilizar EMA-CO, se podría pensar en el uso de EP-EMA (etopósido, cisplatino, metotrexato y actinomicina D) con un periodo de consolidación de 4 ciclos (3). Se ha visto que algunas pacientes con metástasis cerebrales se pueden beneficiar del tratamiento con radiación, además, en algunos casos puede ser necesaria la craniectomía y la quimioterapia intratecal $(3,24)$.

\section{- Tumor de sitio placentario y tumor epitelioide de sitio placentario}

El tratamiento de estos tumores es quirúrgico. Se realiza histerectomía con muestreo de ganglios linfáticos y resección de toda masa sugestiva. En el caso de enfermedad metastásica se brinda tratamiento simultáneo con el régimen EP-EMA hasta 8 semanas luego de la normalización de hCG (20).

\section{- Resistencia al tratamiento}

En el caso de las pacientes con una GTN de bajo riesgo, como se mencionó 
previamente, se puede cambiar el agente utilizado, y si se continúa con resistencia se debe optar por tratamiento con EMACO o MAC(metotrexato, ciclofosfamida y Actinomicina D). Por su parte, en el caso de las pacientes de alto riesgo, si se observa respuesta inadecuada 0 recurrencia luego del uso de EMA-CO, se debe optar por el cabio a EMA-EP (21). Otros tratamientos que se han utilizado son: ACE (dactinomicina, cisplatino y etopósido), VIP (etopósido, isofosfamida y cisplatino), BEP (bleomicina, etopósido y cisplatino) e ICE (altas dosis de isofosfamida, carboplatino y etopósido) (20). En algunos casos, la cirugía para resecar tumores que han probado ser quimioresistentes puede ser curativa (3).

\section{- Seguimiento}

Durante el tratamiento de la NTG, se deben realizar mediciones de hCG cada semana hasta obtener 3 mediciones semanales consecutivas normales. Luego de esto y de la terapia de consolidación, se deben realizar mediciones mensuales de hCG por al menos 1 año para posteriormente seguir con mediciones anuales por 5 años en el caso de las pacientes de bajo riesgo. Para las pacientes de alto riesgo, el esquema de seguimiento varía, siendo mensual por 18 meses, seguido de mediciones cada 6 meses por 2 años, para finalizar con mediciones anuales durante 5 años (20). Además, las pacientes deben utilizar medidas anticonceptivas eficaces (3).

\section{- Pronóstico}

En las NTG de bajo riesgo (estadios FIGO I-III: puntaje <7), la supervivencia promedio generalmente alcanza hasta un $100 \%$; mientras que en las de alto riesgo (estadios FIGO II-III: puntaje $>7$ y estadio
IV), alcanza un 90\%. La inducción con quimioterapia generalmente propicia una reducción de muertes tempranas en pacientes con enfermedad tumoral extensa, sin embargo, la mortalidad tardía se sigue dando en tumores recurrentes y resistentes (3).

\section{PRESERVACIÓN DE LA FERTILIDAD}

Como se mencionó previamente, aproximadamente solo un $1 \%$ de las mujeres con enfermedad molar previa desarrollará embarazos molares en el futuro luego de un tratamiento exitoso. El riesgo es superior en el caso de molas completas (25).

Aproximadamente un $83 \%$ de las pacientes serán capaces de concebir a pesar de haber recibido quimioterapia previa (5). Un artículo de revisión que resumió los datos de 10 centros internacionales, mostró que en pacientes con NTG persistente, la mayoría de ellas presentó embarazos con pronóstico favorable independientemente de los agentes quimioterapéuticos utilizados o la estratificación del riesgo. La incidencia de nacimientos vivos fue de un $76 \%$, y de embarazos de término de un $71,5 \%$. La incidencia de embarazos pretérmino, mortinatos, abortos y anomalías congénitas representó solo en $5 \%, 1,3 \%$, $14 \%$ y $1,3 \%$ respectivamente (2). En pacientes que concibieron a los 6 meses posterior al tratamiento quimioterapéutico, la incidencia de embarazos anormales (abortos, mortinatos y embarazo molar) fue significativamente mayor que para aquellas pacientes que concibieron al año de la terapia. Se aconseja a las pacientes esperar al menos un año para propiciar la 
reparación de $A D N$, evitar la malinterpretación de mediciones de hCG, y evitar los efectos deletéreos de la quimioterapia en la función ovárica y el resultado del embarazo. Pese a esto, los resultados a nivel general siguen siendo favorables y no se recomienda la terminación del embarazo en caso de que el mismo ocurra. Siempre se recomienda un monitoreo cercano en estas pacientes (2).

Un estudio retrospectivo controlado comparó la edad de menopausia en mujeres que recibieron quimioterapia y mujeres que no. En el caso de mujeres que habían recibido quimioterapia, las mismas presentaron la menopausia 3 años previos a lo esperado. Sin embargo, esto no parecía ser causado por insuficiencia ovárica prematura y no presentaba una diferencia clínicamente importante (2). Siempre se puede considerar el tratamiento para fertilidad en pacientes con dificultades para concebir. Como muestran algunos estudios realizados posterior al tratamiento, uno de los principales problemas a la hora de concebir consiste en disfunción sexual (pérdida de libido, dispareunia, problemas de lubricación). Esto se debe abordar con un equipo multidisciplinario. En muchos casos, los síntomas se pueden atribuir en parte a la ansiedad que les genera a las pacientes la posibilidad de otro embarazo desfavorable (2).

\section{CONCLUSIONES}

El manejo de la enfermedad trofoblástica gestacional ha mostrado grandes avances con la medicina moderna, lo cual ha mejorado su curso clínico y disminuido la morbi-mortalidad asociada a esta patología. Es de importancia recalcar la necesidad de una evaluación y diagnóstico oportuno basado en criterios de riesgo y pronóstico, ya que un atraso en el mismo supondría un aumento en el riesgo de una respuesta disminuida al tratamiento; mientras que un diagnóstico oportuno se asocia no solo a un alto potencial de curación, sino a buenas posibilidades de preservar la función reproductiva. Por tanto, y pese a ser una enfermedad infrecuente, el profesional de la salud debe tener conocimiento de la patología y un alto índice de sospecha para evitar errores diagnósticos, terapéuticos y de seguimiento. Sigue siendo necesario propiciar la investigación en casos de neoplasias trofoblásticas gestacionales resistentes a quimioterapia ya que, como se evidenció en la bibliografia, a pesar de que muchas de las pacientes alcanzan curación con tratamientos iniciales, existe un porcentaje de pacientes que presenta enfermedad resistente y que se podría beneficiar de un protocolo de tratamiento más efectivo y estandarizado.

\section{REFERENCIAS}

1. Durón R, Bolaños P. Enfermedad Trofoblástica Gestacional. Med Leg Costa Rica [Internet]. 2018 Marzo [citado 2019 Marzo 04]; 35(1):30-43. Disponible en: http://www.scielo.sa.cr/scielo.php?script=sci arttext\&pid=S1409-00152018000100030\&lng=en

2. Tse KY, Chan KK, Tam KF, Ngan HY. Current management of gestational trophoblastic disease. Obstetrics, Gynaecology \& Reproductive Medicine. 2015 01;25(1):12-21. https://doi.org/10.1016/j.ogrm.2014.10.007 
3. Ngan HY, Seckl MJ, Berkowitz RS, Xiang Y, Golfier F, Sekharan PK, Lurain JR, Massuger L. Update on the diagnosis and management of gestational trophoblastic disease. International Journal of Gynecology \& Obstetrics. 2018 Oct;143(S2):79-85. https://doi.org/10.1002/ijgo.12615

4. Barber E, Soper J. Clinical Gynecologic Oncology [Internet]. 9na ed. Philadelphia: Elsevier; c2018. Gestational Trophoblastic Disease; [citado 2019 Marzo 04]. Disponible en: https://www.sciencedirect.com/search?pub=Clinical\%20Gynecologic\%200ncology\%20\%28Ninth\%20Editi on $\% 29 \&$ cid $=316524 \&$ show $=25 \&$ sortBy=relevance

5. Brown J, Naumann RW, Seckl MJ, Schink J. 15years of progress in gestational trophoblastic disease: Scoring, standardization, and salvage. Gynecologic Oncology. 2017 01;144(1):200-207. https://doi.org/10.1016/i.ygyno.2016.08.330

6. Salani R, Copeland L. Obtetrics Normal and Problem Pregnancies. 7th ed. Philadelphia: Elsevier; c2017. Chapter 50, Malignant Diseases and Pregnancy; p.1070-1073.

7. Parker VL, Tidy JA. Current management of gestational trophoblastic disease. Obstetrics, Gynaecology \& Reproductive Medicine. 2017 Nov;27(11):338-345. https://doi.org/10.1016/i.ogrm.2017.08.004

8. Melamed A, Gockley AA, Joseph NT, Sun SY, Clapp MA, Goldstein DP, Berkowitz RS, Horowitz NS. Effect of race/ethnicity on risk of complete and partial molar pregnancy after adjustment for age. Gynecologic Oncology. 2016 Oct;143(1):73-76. https://doi.org/10.1016/..ygyno.2016.07.117

9. Shaaban AM, Rezvani M, Haroun RR, Kennedy AM, Elsayes KM, Olpin JD, Salama ME, Foster BR, Menias CO. Gestational Trophoblastic Disease: Clinical and Imaging Features. RadioGraphics. 2017 03;37(2):681700. https://doi.org/10.1148/rg.2017160140

10. Johns J, Greenwold N, Buckley S, Jauniaux E. A prospective study of ultrasound screening for molar pregnancies in missed miscarriages. Ultrasound in Obstetrics and Gynecology. 2005;25(5):493-497. https://doi.org/10.1002/uog.1888

11. Mangili G, Lorusso D, Brown J, Pfisterer J, Massuger L, Vaughan M, Ngan HY, Golfier F, Sekharan PK, Charry RC, Poveda A, Kim J, Xiang Y, Berkowtiz R, Seckl MJ. Trophoblastic Disease Review for Diagnosis and Management: A Joint Report From the International Society for the Study of Trophoblastic Disease, European Organisation for the Treatment of Trophoblastic Disease, and the Gynecologic Cancer InterGroup. International Journal of Gynecologic Cancer. 2014 Nov;24(Supp 3):S109-S116. https://doi.org/10.1097/igc.0000000000000294

12. Khanna P. Enfermedad Trofoblástica Gestacional. Revista Médica De Costa Rica Y Centroamérica [Internet]. 2016 [citado 2019 Marzo 04]; 83(618):173-178. Disponible en: http://revistamedicacr.com/index.php/rmcr/article/view/192/177

13. Kaur B, Sebire NJ. Gestational trophoblastic tumours and non-neoplastic trophoblastic lesions: morphology and immunocytochemistry to refine the diagnosis. Diagnostic Histopathology. 2019 02;25(2):53-65. https://doi.org/10.1016/j.mpdhp.2018.12.004

14. Kaur B, Short D, Fisher RA, Savage PM, Seckl MJ, Sebire NJ. Atypical Placental Site Nodule (APSN) and Association With Malignant Gestational Trophoblastic Disease; A Clinicopathologic Study of 21 Cases. International Journal of Gynecological Pathology. 2015 03;34(2):152-158. https://doi.org/10.1097/pgp.0000000000000128

15. Goldstein D, Berkowitz R, Horowitz N. International Manual of Oncology Practice. 1era ed. Cham: Springer; c2015. Diagnosis and Management of Gestational Trophoblastic Neoplasia; p. 501-513. https://doi.org/10.1007/978-3-319-21683-6 21 
16. Froeling FEM, Seckl MJ. Gestational Trophoblastic Tumours: An Update for 2014. Current Oncology Reports. 2014 Oct 16;16(11): 406. https://doi.org/10.1007/s11912-014-0408-y

17. Berkowitz RS, Goldstein DP, Horowitz NS. Management Options of Gestational Trophoblastic Disease. Current Obstetrics and Gynecology Reports. 2013 Nov 10;3(1):76-83. https://doi.org/10.1007/s13669-013$\underline{0065-4}$

18. Biscaro A, Braga A, Berkowitz RS. Diagnosis, classification and treatment of gestational trophoblastic neoplasia. Revista Brasileira de Ginecologia e Obstetrícia. 2015 01;37(1):42-51. https://doi.org/10.1590/so100-720320140005198

19. Gueye M, Ndiaye-Gueye M, Kane-Gueye S, Gassama O, Diallo M, Moreau J. Diagnosis, Treatment and Outcomes of Gestational Trophoblastic Neoplasia in a Low Resource Income Country. Int J MCH AIDS [Internet]. 2016 [citado 2019 marzo 04]; 5(2):112-118. Disponible en: https://www.ncbi.nlm.nih.gov/pmc/articles/PMC5187643/

20. Santaballa A, García Y, Herrero A, Laínez N, Fuentes J, De Juan A, Rodriguez Freixinós V, Aparicio J, Casado A, García-Martinez E. SEOM clinical guidelines in gestational trophoblastic disease (2017). Clinical and Translational Oncology. 2017 Nov 17;20(1):38-46. https://doi.org/10.1007/s12094-017-1793-0

21. Garcia-Sayre J, Castaneda A, Roman L, Matsuo K. Handbook of Gynecology. 1st ed. Cham: Springer International Publishing; c2017. Diagnosis and Management of Gestational Trophoblastic Disease; p. 1-15.

22. Lawrie TA, Alazzam M, Tidy J, Hancock BW, Osborne R. First-line chemotherapy in low-risk gestational trophoblastic neoplasia. . Cochrane Database of Systematic Reviews. 201606 09; Art. No.: CD007102. https://doi.org/10.1002/14651858.cd007102.pub4

23. Couder F, Massardier J, You B, Abbas F, Hajri T, Lotz J, Schott A, Golfier F. Predictive factors of relapse in low-risk gestational trophoblastic neoplasia patients successfully treated with methotrexate alone. American Journal of Obstetrics and Gynecology. 2016 07;215(1):80.e1-80.e7. https://doi.org/10.1016/i.ajog.2016.01.183

24. Patni R. Gestational Trophoblastic Neoplasia: The Role of Surgery Cannot be Undermined!. Indian Journal of Gynecologic Oncology. 2017 Oct 20;15(4): 55. https://doi.org/10.1007/s40944-017-0157-6

25. Eagles N, Sebire N, Short D, Savage P, Seckl M, Fisher R. Risk of recurrent molar pregnancies following complete and partial hydatidiform moles. Human Reproduction. 201507 22;30(9):2055-2063. https://doi.org/10.1093/humrep/dev169 